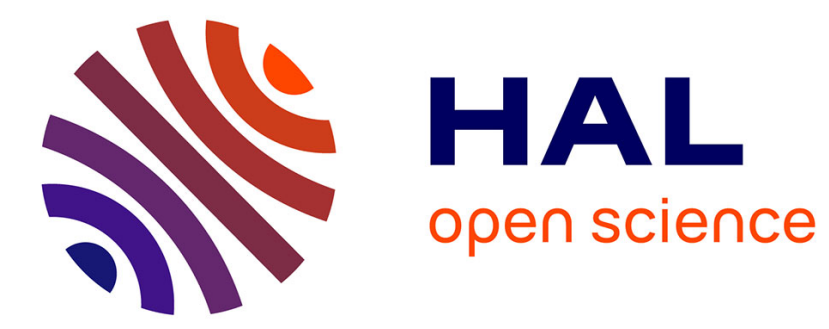

\title{
Controverses autour de la Lutte Contre les Discriminations : introduction 1
}

Johanna Dagorn, Arnaud Alessandrin

\section{To cite this version:}

Johanna Dagorn, Arnaud Alessandrin. Controverses autour de la Lutte Contre les Discriminations: introduction 1. Les Cahiers de la LCD, 2019. hal-02112913

\section{HAL Id: hal-02112913 \\ https://hal.science/hal-02112913}

Submitted on 27 Apr 2019

HAL is a multi-disciplinary open access archive for the deposit and dissemination of scientific research documents, whether they are published or not. The documents may come from teaching and research institutions in France or abroad, or from public or private research centers.
L'archive ouverte pluridisciplinaire HAL, est destinée au dépôt et à la diffusion de documents scientifiques de niveau recherche, publiés ou non, émanant des établissements d'enseignement et de recherche français ou étrangers, des laboratoires publics ou privés. 


\title{
Controverses autour de la Lutte Contre les Discriminations : introduction $^{1}$
}

\author{
Johanna DAgorn \& Arnaud AlesSANDRIN
}

Johanna Dagorn et Arnaud Alessandrin sont chercheuses au LACES (EA 7437) à l'université de Bordeaux et enseignent la sociologie du genre et des discriminations. Tou.te.s deux dirigent les Cahiers de la LCD et coordonnent l'Observatoire Bordelais de l'Egalité. Johanna Dagorn et Arnaud Alessandrin ont récemment mené une série d'enquêtes municipales sur le sentiment de discriminations et sur les déplacements de femmes (2015-2019).

johannadagorn@hotmail.com

arnaud.alessandrin@gmail.com

Depuis leur création, Les Cahiers de la $L C D$ se sont toujours situés à l'articulation entre les revendications individuelles, subjectives, singulières en matière de lutte contre les discriminations, les initiatives politiques et sociales, au niveau national comme local, et les traductions en droit des mouvements de lutte contre les discriminations. C'est dire les difficiles imbrications entre toutes ces sphères et les évidentes contradictions qui en découlent. En vue de leur troisième année d'existence, Les Cahiers de la LCD souhaitent esquisser quelques discussions, en termes de controverses, des nouvelles figures, des nouveaux espaces et des nouvelles déclinaisons de la lutte contre les discriminations, en soulignant les tensions, les paradoxes et les débats qui animent aujourd'hui ce thème.

Presque ontologiquement, la lutte contre les discriminations porte de forts tiraillements. Du point de vue de l'individu, demander la reconnaissance des discriminations vécues ou la protection face à des discriminations potentielles c'est toujours demander une reconnaissance singulière, à l'égard de son identité blessée et, parallèlement, demander une reconnaissance universelle, aux noms du respect de la personne ou de la dignité par exemple. Ces différentes échelles dans la demande de reconnaissance ne sont pas sans créer des tensions entre ce qui est de l'ordre de la reconnaissance «pour soi » et d'une reconnaissance plus universelle. Pour le dire autrement, serais-je prêt à soutenir, au nom des droits humains, des revendications qui ne sont pas les miennes mais qui résonnent avec ces principes? Autre tension perceptible,

\footnotetext{
${ }^{1}$ Pour citer cet article : Johanna Dagorn \& Arnaud Alessandrin, «Controverses autour de la Lutte Contre les Discriminations : introduction », Les cahiers de la LCD, n.9, 2019, 9-18.
} 
toujours du point de vue de l'individu : celle liée à l'endroit de l'identité meurtrie par la discrimination. Si subjectivement cela peut créer des coupures dans les demandes de reconnaissances, il en est de même juridiquement : sur quel·s critère-s dois-je demander réparation?

En creux, la question qui se pose est donc la suivante : sur quel.s autre.s critères de mon identité blessée par la discrimination (l'injure ou le harcèlement) suis-je en mesure de demander réparation, ou du moins une réparation juridique officielle ? L'imbrication des critères et des caractéristiques identitaires s'interroge ici assez nettement (Fassin, 2002).

Juridiquement, les controverses ne sont pas moins actives. Parmi celles qui réapparaissent fréquemment, on compte notamment la question des critères. D'ailleurs, combien y a-t-il de critères au juste (dans le droit pénal, selon le défenseur des droits, selon les décrets d'application etc.) ? Plus encore, ces critères sont-ils efficaces et à quelles conditions ? Au total, y a-t-il trop ou pas assez de critères ? À bien regarder les chiffres des dépôts de plainte (5 213 réclamations en 2016 auprès du défenseur des droits), toutes discriminations confondues, nous sommes en mesure de souligner l'hypo-efficacité du droit en la matière. Non que les reconnaissances et les réparations juridiques rendues ne soient pas importantes, pour les victimes comme pour l'application générale du principe de non-discrimination, mais plutôt, que deviennent toutes les victimes qui ne sont pas parvenues porter plainte ou à passer en justice ? C'est cet écart entre l'augmentation tendancielle du nombre de critères et la stagnation des taux de condamnation qu'il convient également d'interroger, au regard de l'accès aux droits, aux stratégies collectives d'accompagnement des victimes, au regard des dispositifs associatifs et administratifs disponibles, au regard de leur (sous) financement etc. Bref, comment lutter contre l'hypo-efficacité du droit en la matière (qu'il s'agisse de dépôt de plainte, d'instruction des dossiers et ou de peines prononcées, de recours, d'illégalité et ou de dommages-intérêts) ? La réponse en vue d'un élargissement de l'assiette de la reconnaissance, est-elle toujours à l'accroissement du nombre de critères ? D'ailleurs, les critères diffèrent tellement d'un pays à l'autre, que seuls cinq critères sont mesurés au niveau européen : le sexe, l'origine ethnique, la religion ou les convictions, l'âge, le handicap et l'orientation sexuelle (selon l'eurobaromètre depuis 2009). D'un point de vue victimaire, il est fort à parier que la présence d'un critère qui résonne avec les épreuves endurées n'est pas anodine dans le processus de reconstruction, de reconnaissance et de réhabilitation. On se souvient du critère de «transphobie » qui, en 2012, dans une forme première, avait été reconnue sous les traits d'une discrimination à l'encontre de «l'identité sexuelle ». Ne signifiant pas grand-chose, ni dans une perspective sociologique, ni eu égard aux textes juridiques européens, ni - et c'est peut-être ce qui anime la lutte contre les discriminations - auprès des personnes victimes de transphobie, il aura fallu attendre 2016 pour que le terme de «discrimination à l'encontre de l'identité de genre de la personne » fasse son entrée dans le droit français. Dans cette perspective, la reconnaissance juridique, même hypothétique, est passée par la création d'un nouveau critère (Alessandrin, 2016).

On pourrait faire le constat que, dans le paysage actuel, ces nouvelles terminologies explosent. Elles tendent à circonscrire des discriminations non encore identifiées par le droit ou, plus précisément, non identifiées comme telles. Si l'on pense, par exemple, à 
l'islamophobie (Asal et Mohammed, 2014), la «gérontophobie » (Maisondieu, 2003) ou à la grossophobie (Deydier, 2017), nous serions tentés de croire que les critères de « religion réelle ou supposée » ou d' «apparence» suffisent. Or si les discriminations sont des expériences incorporées, expérimentées, donc sensibles, ces termes ne semblent pas du tout être en capacité de recouvrir les demandes de reconnaissances des personnes victimes ou potentiellement victimes.

Trop floues, trop vagues, pas assez spécifiques aux populations et aux situations auxquelles elles sont censées s'adresser, ces terminologies («apparence »...) sont débordées.

Il est tout aussi intéressant de souligner qu'à l'opposé de ces revendications (plus que légitimes au vu des chiffres des discriminations encourues par ces populations) ${ }^{2}$, le vocabulaire de la discrimination est aussi employé par ceux-là mêmes qui combattent la lutte contre les discriminations. N'avons-nous pas récemment entendu les termes de « racisme antiblanc » dans de nombreux médias ou d' «hétérophobie »? Or en accord avec Éric Fassin (2017), «le racisme anti-Blanc n'existe pas, pour les sciences sociales, ça n'a pas de sens ». C'est en effet nier les discriminations directes et systémiques à l'embauche, notammen vécues par les personnes racisées. Pour le dire autrement, la généralisation du champ lexical de discrimination doit aussi nous interroger sur les récupérations, les instrumentalisations, les horizontalisations abusives, qui parsèment la lutte contre les discriminations; d'autant plus que l'opposition aux demandes «particularistes » s'étend aujourd'hui sur plusieurs fronts, que ce soit au nom d'une humanité universelle et indivisible ou au nom d'une remise en cause trop massive et trop brutale des habitudes (Barberis, 2019 ; Wagner et Bodenan, 2018).

Enfin, du point de vue politique, les crispations autour de ce terme sont également bien présentes et ces dernières années ne semblent pas avoir assouvi les espoirs en matière de réduction des discriminations. Si des campagnes de communications (comme les LGBTphobies à l'école) et des plateformes de signalement en ligne (comme pour les discriminations sexistes par exemple) ont été lancées, si la DILCRAH (direction interministerielle de lutte contre les discriminations racistes, antisémites et homophobes) lance des appels à projets annuels, les impulsions fortes semblent délaissées, à la charge de quelques régions et municipalités qui travaillent sur ces questions, marquant là l'isolement de la lutte contre les discriminations à quelques «bonnes volontés » ou encore à quelques « réactions à des cas médiatisés ».

Pour discuter ces différents éléments, et pour ouvrir encore d'autres pistes, ce présent volume des Cahiers de la LCD accueille plusieurs textes d'horizons différents. Le premier texte, de Milena Doychteva, revient sur les usages des catégories territoriales (Doytcheva, 2016) en matière de lutte contre les discriminations et met en perspective les notions « d'exclusion » et de «discrimination». La question, que nous pouvons reformuler à ses côtés, est la suivante : réduire la lutte contre l'exclusion à des territoires politiques de la ville, n'est-ce pas en réduire

\footnotetext{
${ }^{2}$ Pour exemple récent, dans nos dernières enquêtes sur les discriminations et harcèlements subis par les femmes dans l'espace public dans cinq villes françaises $(\mathrm{N}=10052)$, les publics ayant «l'indice de climat urbain » le plus dégradé étaient, aux côtés des femmes employées et ouvrières, les femmes vues comme « grosses », les femmes «trans» et les femmes racisées (prioritairement les femmes voilées et roms). Lire à ce propos, Dagorn J. et Alessandrin A. (2018), «Discriminations et harcèlement des femmes dans la ville : une analyse intersectionnelle », in (Navarre M. et Ubbiali G., dir.), Le genre dans l'espace public, L'Harmattan, pp. 143-163.
} 
la portée plus générale ? En parallèle, circonscrire l'exclusion à ces mêmes territoires ne revient-il pas, une nouvelle fois, à nier les réalités des phénomènes racistes et discriminatoires ? En proposant de «déterritorialiser l'exclusion» et de «re-territorialiser le racisme et les discriminations », la chercheuse pose frontalement la question des politiques publiques de lutte contre le harcèlement et, au-delà de leur efficacité, la question de leur hypocrisie.

La poursuite de ce volume nous amène à une autre interrogation, celle des « espaces » de la lutte contre les discriminations. Si l'actualité récente a beaucoup mis en lumière les espaces publics comme des théâtres de l'expérience discriminatoire (antisémite, sexiste ou homophobe), internet reste à n'en point douter le lieu d'explosion des phénomènes d'injure et de harcèlement à caractère discriminatoire. À travers leurs enquêtes sur le cyberharcèlement (Couchot-Schiex, Moignard, et Richard, 2016), Sigolène Couchot-Schiex, Benjamin Moignard et Gabrielle Richard interrogent l'expérience du web chez les jeunes «minoritaires» (au sens des minorités de genre et de sexualité). Face à la description spontanée d'un espace numérique hostile, les chercheuses et chercheurs mettent en avant les résistances qu'opèrent les jeunes utilisateurs LGBT+ face aux injonctions hétérosexistes et aux attaques discriminatoires. Dans un contexte plus général de déplacement de la lutte contre les discriminations à de nouveaux espaces, virtuels notamment, la question des ressources et des moyens dont disposent les individus pour faire face à ces attaques, interroge fondamentalement l'engagement des institutions (scolaires, sanitaires etc.) dans ces nouvelles réalités.

Nous poursuivons notre volume avec un texte d'Arthur Vuattoux qui se penche, comme il a déjà eu l'occasion de le faire pour sa thèse (Vuattoux, 2016), sur le traitement juridique des mineurs en France, en particulier les mineurs, là encore, « minoritaires ». L'exercice offert par Arthur Vuattoux pose ici la question du «lieu» de la défense et de la reconnaissance. À l'image des enquêtes sur l'accueil des femmes victimes de violences dans les commissariats en France (enquête VIRAGE, 2017), le sociologue met en relief que des espaces du droit peuvent s'apparenter à des espaces de discriminations à l'encontre de corps et d'identités « minoritaires ». À travers l'exemple des jeunes filles roms, l'auteur parvient à montrer la surpénalisation de certains profils, dans une dynamique intersectionnelle qui croise les questions de genre et les questions de classes sociales. Intitulé «Corps majoritaire, corps minoritaire/réponses judiciaires et traitement différentiel des corps dans la justice des mineurs française », cet article a aussi le mérite de rappeler que les identités ne sont pas que des étiquettes qui valsent d'un particularisme à un autre, mais qu'elles ont aussi des encrages corporels qui traduisent, à leurs corps défendant, des stéréotypes pas toujours objectivables, mais bel et bien présents dans les processus discriminatoires.

Un dernier article compose ce volume, celui de Yoann Lopez autour des notions de «mémoire » et de «lutte contre les discriminations ». Issu d'une recherche-action menée à l'échelle d'une ville (Bordeaux) et d'une thèse en sociologie sur cette même question (Lopez, 2012), cet article retrace les liens entre des politiques mémorielles et des politiques antidiscriminatoires et interroge : «en quoi une différence de traitement mémorielle peut-elle être discriminatoire ? ». Après avoir rappelé les imbrications historiques et subjectives qui se tissent encore entre enjeux mémoriels et enjeux de lutte contre les discriminations, le sociologue dresse un panorama des logiques mémorielles (à l'échelle municipale notamment) 
en direction des minorités concernées. Il est à noter que les questions de mémoire - et au-delà des mémoires de l'esclavage ou de l'holocauste - sont aujourd'hui mobilisées par de nombreuses minorités non seulement pour « redécouvrir » un passé commun mais aussi pour le préserver.

Dans ce volume, nous accueillons aussi un texte d'Abdellatif Chaouite, rédacteur en chef de la revue Écarts d'identité et intitulé «L'autre de l'autre ». Il s'agit d'une carte blanche laissée à l'auteur, son regard sur les discriminations, l'interculturalité (Chaouite, 2016) et l'altérité dans le monde actuel, et son rôle en tant que directeur d'une revue qui a pour horizon, comme la nôtre, la lutte contre les discriminations.

Enfin, nous concluons ce volume par deux restitutions de colloques. La première reprend et synthétise les rencontres organisées par le défenseur des droits le mardi 22 janvier 2019. Intitulé « La multiplication des critères de discrimination renforce-t-elle l'effectivité du droit de la non-discrimination », il est repris et résumé par Julie Voldoire. La seconde rend compte des échanges qui se sont déroulés les 23 et 24 avril 2018 à l'université de Bordeaux lors du colloque «Quel est le rôle de la ville dans la lutte contre les discriminations ? ». Réalisée par Elsa Koerner, cette synthèse fait également écho à notre premier numéro de revue : « La ville face aux discriminations » (Dagorn, Alessandrin et Charaï, 2016).

Évidemment, ces thématiques ne sauraient être exhaustives face à l'effervescence des discussions et controverses qui traversent le champ de la LCD. C'est aussi pour nous l'occasion de souligner combien de thématiques Les Cahiers de la LCD ont parcouru en 3 ans d'existence et, en négatif, combien d'autres restent à traiter.

\section{Bibliographie}

Alessandrin A. (2016), «La transphobie en France : insuffisance du droit et expériences de discrimination », Cahiers du Genre, 60, 1, pp : 193-212.

Asal H. et Mohammed M. (2014), «Islamophobie en France : formes, définitions et mesures », in (Poinsot M. et Weber S., dir.), Migrations et mutations de la société française, La Découverte, pp. 316-323.

Barberis I. (2019), L'art du politiquement correct, PUF.

Chaouite A., Ferhat B. et Righi F. (2016), L'expérience interculturelle dans l'intervention sociale, L'Harmattan.

Couchot-Schiex S., Moignard B. et Richard G. (2016), Cybersexisme : une étude sociologique des établissements scolaires françiliens, Centre Hubertine Auclert.

Dagorn J. et Alessandrin A. (2018), «Discriminations et harcèlement des femmes dans la ville : une analyse intersectionnelle », in (Navarre M. et Ubbiali G., dir.), Le genre dans l'espace public, Harmattan, pp. 143-163.

Dagorn J., Alessandrin A. et Charaï N. (dir.), (2016), «La ville face aux discriminations », Les Cahiers de la LCD, vol. 1.

Deydier G. (2017), On ne naît pas grosse, Goutte d'or éditions. 
Doytcheva M. (2016), «Usages et mésusages des catégories territoriales : le risque d'un retournement idéologique des causalités », Les Cahiers de la LCD, vol. 1, pp. 58-74.

Fassin D. (2002), «L'invention française de la discrimination », Revue française de science politique, $\mathrm{n}^{\circ} 4$, vol. 52, pp. 403-423.

Lopez Y. (2012), «Retour sur la construction identitaire noire en France : la question raciale face à l'universalisme républicain », in (Cortesero R., dir.), La banlieue change !, Éditions Le Bord de l'eau, pp. 119-122.

Maisondieu M. (2003), «Vieillir en famille : une situation cornélienne », Cahiers critiques de thérapie familiales et de pratiques de réseaux, $\mathrm{n}^{\circ} 31$, pp. 99-110.

Vuattoux A. (2016), «Le traitement institutionnel d'une minorité par la justice en Île-deFrance : le cas des "jeunes filles roumaines" », Revue femmes et droit, n 3 , vol. 28, pp. 646666.

VIRAGE (2017), Premiers résultats sur les violences sexuelles, Document de travail $n^{\circ} 229$, Ined.

Wagner G. et Bodenan M. (2018), «Les sorts des particularismes », Lignes, $\mathrm{n}^{\circ}$ 57, vol. 3, pp. 49-57. 
